\title{
LE MOMENT BERR
}

\author{
Jacques REVEL
}

1. À peine avais-je proposé un titre provisoire aux organisateurs de ce colloque, je m'en suis repenti. J'avais pensé ruser aussi, et jouer la prudence en recourant à une fausse interrogation comme: "Y a-t-il eu un moment Berr? » Je ne suis, à dire vrai, pas sorti de cette incertitude et pas même assuré que le terme de «moment ", que j'ai fini par retenir, soit bien choisi. Et ce pour trois raisons au moins.

La première, c'est que Berr lui-même eût sans nul doute récusé le mot et l'idée. Il l'a écrit de façon tout à fait explicite dans La Synthèse en histoire (1911), alors qu'il réfléchissait sur les formes de l'innovation (qu'il appelle «l'invention») dans la connaissance scientifique: "La maturité détache le fruit de la raison de plusieurs côtés à la fois. C'est là, sans doute, ce que Taine entendait, de façon vague, par le moment; et c'est du moins ce qu'enferme de juste le terme imprécis de Taine ${ }^{1}$. » Pour euphémisée qu'elle soit, dans le style inimitable qui est celui de Berr, la réserve est explicite : il n'eût pas aimé que l'on parlât d'un moment Berr. L'objection n'est sans doute pas décisive; elle n'est pas non plus négligeable.

La seconde raison renvoie à la chronologie d'une vie et d'une œuvre qui couvre une période d'une extrême longueur: plus d'un demi-siècle, voire soixante ans en comptant large, pendant lesquels la pensée de notre auteur n'a pas cessé de revenir sur ses traces sans connaître de rupture ni même d'inflexion marquée. Non seulement La Synthèse de 1911 a été republiée encore un an avant la mort d'Henri Berr, en 1953, quasi à l'identique, mais tous ses lecteurs savent que l'on retrouve chez lui, reprises d'une décen-

1. Henri Berr, La Synthèse en histoire, Paris, 1911. Pour plus de commodité, je me réfère à la dernière édition, Paris, A. Michel, 1953, p. 112. 
nie à l'autre, des premiers débuts de la revue aux années du Centre, non seulement des formules mais parfois des phrases entières. Cette continuité de la pensée, étirée par la grâce d'une longévité exceptionnelle et dictée par une fidélité à soi-même non moins remarquable, tout cela suggère un «moment » singulièrement prolongé et distendu. Sauf à poser que la personnalité intellectuelle de Berr a dominé le premier $\mathrm{XX}^{\mathrm{e}}$ siècle tout entier - ce qui pourra, malgré tout, apparaître exagéré - est-il seulement possible de cerner un tel moment?

J'en viens à ma troisième raison. Henri Berr était porté par un optimisme de la raison, qui a sous-tendu à la fois ses conceptions scientifiques et ses multiples entreprises. Du volontarisme intellectuel qui a traduit cette confiance, la rencontre qui nous réunit autour de son œuvre a donné nombre de témoignages. Comme chez Durkheim, son presque contemporain, il a revêtu une double dimension indissolublement scientifique et éthique, mieux, éthico-politique. Berr lui-même a aimé réfléchir publiquement sur le bilan de son action, et il l'a souvent fait, convenons-en, avec un certain triomphalisme. Et pourtant il a aussi laissé, plus rarement, percer le sentiment d'un certain découragement, voire l'aveu d'un échec derrière la réussite apparente. Ses œuvres ont été des succès, son œuvre n'a pas été véritablement reçue. Il l'a écrit dans l'un de ses derniers textes, en appendice à l'ultime réédition de La Synthèse :

«Beaucoup d'idées ont cheminé, qui inspiraient en 1900, la Revue de synthèse historique, en 1911 le présent livre, depuis 1920 'L'Évolution de l'humanité"; mais notre conception de la synthèse scientifique n'a été, nous l'avons dit, ni pleinement appliquée, ni même sérieusement discutée. Il semble que de cette "Synthèse en Histoire", on ait apprécié le détail et négligé l'essentiel ${ }^{2}$."

Bel exercice de lucidité, de la part d'un homme que l'on peut parfois soupçonner de s'être payé de mots... Il reste que ce bilan morose, cette révvaluation à la baisse ne viennent pas non plus conforter mon projet. $\mathrm{Si}$ l'on en croit l'intéressé lui-même, il n'y aurait jamais véritablement eu de «moment Berr».

Me voici donc bien mal parti. Je voudrais pourtant tenter de défendre mon projet et même tenter de l'illustrer, en commençant par préciser ce que j'entends par «moment» - et qui, d'ailleurs, n'est pas original. Ce sont deux aspects, pour l'essentiel. Le premier propose un état des lieux et voudrait tenter de caractériser une conjoncture intellectuelle. Je ne suis

2. lbid., p. 307-308. 
pas de ceux qui estiment (mais existent-ils ?) que Berr a été un théricien majeur. Il me paraît évident, en revanche, qu'il a voulu et qu'il a su donner une formulation à un moment particulier du débat français et par moments européen. Il l'a fait à côté d'autres, avec d'autres, mais à sa manière qui est clairement reconnaissable. Mais il a fait plus : il a su prendre appui sur ce débat, il s'est employé à l'organiser à travers les projets, tout à la fois fortement personnels et toujours collectifs, dont il a été l'animateur dès le début du siècle. Parler de «moment" revient, en ce sens, à reconnaître que la politique scientifique dont il s'est voulu le promoteur, en marge de toutes les institutions régulières du savoir académique ${ }^{3}$, n'a de sens que dans le cadre d'une certaine histoire — ou plutôt d'un agencement d'histoires dont il a longuement analysé l'écheveau et dont la plus récente, celle dont Berr était le contemporain, lui paraissait appeler impérieusement la synthèse générale des connaissances. Je n'ai pas, en ce sens, le sentiment de faire violence à sa pensée en la situant historiquement. Le second aspect pourrait être plus contentieux et il eût sans nul doute rencontré plus de réticences, comme je ne doute pas qu'il en suscite aujourd'hui. Il suggère en effet une manière de périodisation au sein d'une activité que Berr a lui-même placée, au prix d'incessants retours et ressassements, sous le signe de la continuité. Je voudrais ainsi suggérer que la période de la réussite publique la plus visible, celle du Centre de synthèse à la fin des années 20 et dans les années 30 , n'est pas forcément la plus significative du projet berrien dont je situerai le centre de gravité plus tôt, dans la quinzaine d'années qui précèdent la Première Guerre mondiale. Non seulement parce que c'est le temps de son ouvre la plus personnelle et de celui de son livre le plus durable, mais tout autant parce que c'est alors que Berr a mis en place, autour de la première Revue de synthèse historique, le programme et les modalités d'une collaboration scientifique collective qui est inséparable de son projet intellectuel; c'est aussi le moment où il en contrôle le mieux, personnellement, les moyens et les effets - mieux, peut-être, que cela ne sera le cas vingt-cinq ans plus tard, quand la lourdeur institutionnelle du Centre et la multiplication des protagonistes paraîtra parfois compliquer sa tâche d'organisateur.

2. Il importe donc de partir du débat au sein duquel le projet d'Henri Berr trouve sa place. Il convient en ce point de rappeler que sa formation, ses préoccupations, ses références ont d'abord été celles d'un philosophe.

3. Sur ce point, cf. Giuliana GEMELLI, «Communauté intellectuelle et stratégies institutionnelles; Henri Berr et la fondation du Centre international de synthèse », Revue de synthese, 2, 1987, p. 225-259. 
Sur cette dimension du personnage, reconnaissons que nous savons fort peu de choses parce qu'il n'a guère retenu l'attention des spécialistes ${ }^{4}$. En l'absence de toute compétence, je me garderai bien de me substituer à ceuxci. Mais, faute de mieux, la lecture des textes «philosophiques» de Berr — en particulier, celle de la thèse de 1899, L'Avenir de la science ${ }^{5}$, qui apparaît clairement comme une première élaboration de son système me parait suggérer trois constats dont j'espère qu'ils ne heurteront personne.

Le premier est qu'il s'agit là, à l'évidence, d'une construction éclectique. C. B. Krol nous a proposé de voir en Berr un mystique pascalien. En l'écoutant et en lisant Berr, j'aurais penché plutôt pour un spinoziste optimiste (ce qui est, peut-être, contradictoire dans les termes). Mais peu importe. On trouve tout chez notre auteur: le néo-kantisme qui est le fonds commun de sa génération intellectuelle; un hégélianisme qui paraît avoir résisté à sa propre critique des philosophies de l'histoire; un positivisme spiritualisé, dont il n'est pas, tant s'en faut, le seul représentant au tournant du siècle ; un vitalisme dans lequel on pourra être tenté de retrouver quelque influence bergsonienne, au moins dans le lexique. Une citation parmi beaucoup d'autres possibles peut servir à illustrer ce patchwork, et suggère au passage la vanité de toute recherche de filiation:

«L'histoire, telle que nous la comprenons [...] peut donner à tant d'êtres, à tant de jeunes surtout, en désarroi intellectuel, la joie d'embrasser le temps, de comprendre la vie, de se dépasser, pour ainsi dire, en situant l'individu dans l'Humanité et l'Humanité elle-même dans le réel ${ }^{6}$.»

Il n'est probablement pas très utile de perdre trop de temps au jeu du "de qui est-ce? ». Il n'y a d'ailleurs rien là de très choquant ni même d'original. L'éclectisme de Berr, dont on trouverait d'autres exemples chez Fouillée et chez bien d'autres, est une des solutions philosophiques inventées en cette époque de crise, comme l'a bien montré J.-L. Fabiani ${ }^{7}$.

4. C'est d'ailleurs là un problème récurrent des (rares) travaux consacrés à Berr : historiens, philosophes, sociologues, aucun corps de métier ne le revendique explicitement comme l'un de ses membres, et il se trouve ainsi absent de la plupart des analyses consacrées à la période, ou bien il y figure en position marginale. Pour la philosophie, cf. J.-L. FABIANI, Les Philosophes de la République, Paris, Minuit, 1988.

5. H. BERR, L'Avenir de la philosophie: esquisse d'une synthèse des connaissances fondée sur l'histoire, Paris, 1898.

6. Op. cit. supra n. 1, p. 308.

7. Notons que l'imputation d'éclectisme n'est pas seulement produite par notre point de vue, à un siècle de distance. Elle est déjà contemporaine : ainsi dans la brève note, plutôt sympathique d'ailleurs, que l'historien Gabriel Monod consacre à L'Avenir de la philosophie dès après sa publication: «Par moments, la synthèse de M. Berr nous apparaissait comme 
Le projet philosophique de Berr n'est pas seulement éclectique, il mêle des genres qui étaient traditionnellement séparés. Cela est vrai dans son expression personnelle, qui juxtapose parfois dans une même page le registre de la philosophie classique, le compte rendu critique, l'effusion personnelle (rappelons-nous la montée mystique au Ballon d'Alsace, qui clôt L'Avenir de la philosophie), le militantisme organisateur. De cet aspect, qui n'est pas si trivial, tous les lecteurs de Berr sont familiers. Mais cela se vérifie tout autant dans la manière dont il a conçu son rôle culturel, et cela, semblet-il, très tôt. Ce n'est pas seulement après les deux échecs de sa candidature au Collège de France $(1904,1913)$, et par une sorte de compensation, mais bien plus tôt que Berr a pensé qu'il était nécessaire de sortir du cadre strict de la République des professeurs et du débat français. La conviction que la nécessaire synthèse des connaissances relève d'une politique volontariste et organisatrice est présente aux origines de la Revue de synthèse historique, bien entendu, mais elle se lit déjà dès les premières œuvres, L'Avenir de la philosophie et même Vie et science. Lettres d'un vieux philosophe strasbourgeois et d'un étudiant parisien, publié en 1894 alors que notre auteur venait à peine de passer la trentaine. On peut, sans forcer le trait, considérer que les entreprises successives de Berr n'ont été qu'une manière de mettre en œuvre de façon de plus en plus large et plus diversifiée, selon des agencements toujours plus complexes, le projet d'une mise en regard systématique des savoirs inscrits dans une perspective historique.

Il reste que, si ouverts qu'aient été ses intérêts, si boulimiques ses lectures, si démesurées ses ambitions, la posture intellectuelle de Berr est demeurée de nature classiquement philosophique. C'est celle d'un homme soucieux de construire un cadre épistémologique - ou, à tout le moins, un cahier des charges commun et explicite - pour la confrontation de démarches scientifiques qu'il ne pratique pas lui-même, qu'il s'agisse de l'histoire, des diverses sciences sociales, des sciences de la nature ou de l'histoire des sciences. Il reprend sans hésiter à son compte une division classique du travail, particulièrement ancrée dans la tradition académique et intellectuelle française, et qui confère au philosophe le privilège de penser les raisons de pratiques qui lui demeurent, pour l'essentiel, extérieures. On sait que cette tradition conserve de beaux restes aujourd'hui.

un éclectisme d'un nouveau genre; à d'autres, elle nous faisait l'effet d'un monisme hégélien assez original où tout absolu métaphysique s'évanouissait pour ne laisser subsister qu'un universel devenir, où la forme et le mouvement étaient les seules réalités, en un mot où la philosophie devenait science et histoire », «Bulletin historique », Revue historique, 70, 1899, p. 99. 
3. Ce n'est donc pas du côté du propos philosophique de Berr que je rechercherai sa plus grande originalité. Il me paraît conforme tout à la fois à un certain état de la discipline, aux questions qu'elle se pose au tournant du siècle et à ses manières de faire. Ce n'est pas non plus de ce côté, me semble-t-il, qu'il convient de chercher la part la plus importante de son œuvre (dans la perspective qui est la mienne ici; il va de soi que les choses seraient différentes si je m'intéressais plus spécifiquement à l'œuvre ou à la biographie d'Henri Berr). Cette originalité et cette importance, ce qui rend ce projet topique au moment où il est énoncé, je les trouve plutôt dans la proposition qui est faite - et qui va être, dans une large mesure, réalisée - d'un modèle de collaboration scientifique qui associe autour d'une conception de la connaissance et de son histoire, l'organisation d'une sociabilité, l'invention de formes du travail, la construction d'un mode de relations et de confrontations entre les disciplines.

Partons du plus visible et, peut-être, du plus extérieur. La création d'espaces de sociabilité scientifique est au cœur des entreprises auxquelles Berr s'est attaché tout au long de sa vie. Elle a pris des formes successives, dont le colloque qui nous réunit a examiné quelques-unes. Ces formes se sont succédé, accumulées, déformées en cours d'expérience; certaines ont été abandonnées, d'autres remployées; elles n'ont cessé de proliférer, de se faire plus complexes, peut-être aussi plus floues à mesure qu'elles se voulaient davantage englobantes. Il reste que dans la vocation organisatrice de Berr (et son indéniable talent en la matière), il faut voir bien autre chose qu'une lubie personnelle - dont Bloch et Febvre l'ont parfois raillé, sans trop d'indulgence. Il me paraît avoir, au contraire, répondu à une série de problèmes très clairement posés, et de façon pressante, entre les dernières années du XIX ${ }^{e}$ siècle et la Première Guerre mondiale.

Dans l'Université française refondée par la $I I I^{\mathrm{e}}$ République, l'accent a été mis sur la spécialisation et la professionnalisation, par réaction contre la définition généraliste et le primat rhétorique, désormais considéré comme abusif et dommageable, de l'ancien système universitaire. Fritz Ringer a récemment montré comment des mouvements contraires avaient valorisé en France une évolution qui, au même moment, paraissait menacer dans le monde allemand l'unité de la Bildung ${ }^{8}$. Il reste que, pour être globalement fondé, le constat ne doit pas cacher les inquiétudes qui ont accueilli la mise en place de la Nouvelle Sorbonne. Inquiétudes idéologiques : elles

8. Fritz RINGER, Fields of Knowledge. French Academic Culture in Comparative Perspective, Cambridge-Paris, 1992. Sur ces problèmes, l'étude de référence reste celle de G. WEIsz, The Emergence of Modern Universities in France (1863-1914), Princeton, 1983. 
sont bien connues et s'expriment surtout du côté de l'extrême droite maurassienne, au nom des droits imprescriptibles de la culture générale et de la tradition des humanités ${ }^{9}$. Mais elles ne sont pas les seules à s'exprimer. $\mathrm{Ne}$ voit-on pas toutes les disciplines, les anciennes (comme l'histoire ou la philosophie), mais aussi et surtout les nouvelles (la géographie, la psychologie, l'économie, la sociologie) s'organiser en cantonnant leur territoire? Elles se dotent d'associations professionnelles, de revues spécialisées, de congrès nationaux et, bientôt, internationaux. Aux yeux de beaucoup, le risque paraît réel d'une compartimentation, sans doute inévitable mais aussi dommageable et, à terme, menaçante de l'espace intellectuel tel que le conçoit l'Université. Face à ce risque, les réactions sont multiples. On pourrait, sans forcer les choses, poser que Durkheim, par ailleurs partisan déclaré de la spécialisation et de la professionnalisation des études, a voulu ouvrir un espace commun de discussion critique avec la création de L'Année sociologique (1898). L'originalité de la conception d'Henri Berr, et des stratégies qu'elle a engendrées, c'est d'avoir tenté d'organiser un espace similaire hors de l'Université même si, au départ au moins, c'est surtout avec des universitaires qu'il l'a fait ${ }^{10}$.

À lui comme à plusieurs de ses contemporains, le problème scientifique est apparu, en outre, aggravé par un autre facteur de division, qui lui a paru requérir d'autant plus l'affirmation d'une solidarité entre les hommes de savoir. Les premières interventions de Berr, ses premiers livres puis la fondation de la revue sont exactement contemporains de l'affaire Dreyfus. Recréer les conditions d'une communauté de langage et la possibilité d'échanges répond donc aussi à une urgence politique, dont témoigne l'interrogation de 1901: «Peut-on refaire l'unité morale de la France " ? " Henri Berr, Christophe Prochasson le montre ici, est introuvable du côté de la pensée socialiste. On le retrouve, en revanche, en compagnie de ceux qui sont convaincus de la vocation de la science à réunir plutôt qu'à diviser : elle est conciliatrice parce qu'elle est œuvre de raison et de progrès. Mais, de nouveau, l'Université lui paraît trop étroite pour mettre en œuvre un tel programme. Il trouve donc sa place au milieu des initiatives privées, nombreuses au tournant du siècle, qui veulent à la fois socialiser la connaissance et fonder sur cette circulation

9. Voir, pour prendre un exemple moins souvent invoqué que celui d'Agathon et plus significatif pour notre propos, le volumineux pamphlet de Pierre LASSERrE, La Doctrine officielle de l'Université, Paris, 1912.

10. On sait à quel point Berr, tout en reconnaissant les mérites scientifiques de Durkheim et de L'Année sociologique, est resté réservé devant ce qu'il percevait comme trop universitaire et trop sectaire, trop professionel aussi dans le mouvement sociologique.

11. H. BERR, Peut-on refaire l'unité morale de la France?, Paris, A. Colin, 1901. 
une communauté civique ${ }^{12}$. L'Affaire retombée, cette préoccupation ne cesse pas pour autant. Elle se transforme puisqu'il s'agit désormais de conserver les solidarités qui avaient rapproché une large part des «intellectuels " sous la pression de l'événement. On peut lire le premier des bilans décennaux publiés par Berr, le célèbre texte " Au bout de dix ans », comme une revendication de l'efficacité de son action à travers la Revue de synthèse historique : face aux forces qui la menaçaient du dehors, mais aussi face aux tensions surgies au lendemain de la crise ${ }^{13}$.

Qu'il ne se soit pas seulement payé de mots, la réussite de la revue suffirait à l'attester: la revue, mais tout autant son mode de fonctionnement. Lucien Febvre, qui y a fait ses premières armes d'historien, a rappelé beaucoup plus tard ce qu'elle avait été pour lui dans ses années de formation à l'École normale :

«... Cette revue qui proclamait et réalisait le dessein de réunir pour une œuvre de synthèse efficace, des historiens et des archivistes, des géographes et des ethnologues, des linguistes, des économistes et des philosophes, tous fraternellement unis dans le souci de l'œuvre commune - cette Revue qui, là où les autres ne savaient que distiller l'ennui, installait l'enthousiasme et l'espérance $[\ldots]$, quelle libération et quelle joie ${ }^{14}$.»

Objectera-t-on qu'il s'agit là d'un témoignage tardif, porté à plus d'un demi-siècle de distance, et d'abord destiné à honorer le vieux maître ? Outre qu'il existe d'autres preuves de l'attachement de Febvre au premier Berr - La Synthèse en histoire n'est-il pas «le seul livre de méthode que j'aie jamais fait lire à mes étudiants "? -, tout atteste que la Revue de synthèse historique a été, dès ses premières années, capable de rassembler des participations tout à la fois nombreuses et diversifiées. Il s'agit, après tout, d'une initiative individuelle et quasi privée, qui ne disposait au départ d'aucune légitimité institutionnelle ni scientifique forte, qui ne se référait à aucune orthodoxie connue ou reconnaissable. Or avant même d'être en mesure de constituer ses propres réseaux, la nouvelle publication s'est montrée capable d'attirer plusieurs catégories de collaborateurs: les uns, probablement passifs, sont les grands noms (de moins grands aussi parfois)

12. Je renvoie ici aux récents travaux de Christophe Prochasson sur les intellectuels au tournant du siècle, en particulier sur la naissance de la Revue de métaphysique et de morale ainsi que sur l'École des hautes études en sciences sociales, sur Simiand.

13. H. BERR, «Au bout de dix ans », Revue de synthèse historique, 61, 1910, p. 1-13.

14. Lucien FeBvrE, «De la Revue de synthèse aux Annales: Henri Berr, ou un demi-siècle de travail au service de l'histoire $»$, Annales ESC, 1952, p. 290. Notons que les termes ne sont pas très différents de ceux que le même Febvre emploie pour évoquer, dans ces mêmes années, sa découverte de L'Année sociologique. 
dont Berr, lecteur boulimique, a fait traduire et publier des textes pour nourrir le débat français, par une politique de «rattrapage»; viennent ensuite ceux qui sont ses proches et qui, le plus souvent, le resteront, de Paul Lacombe au Dr Jankélévitch; puis les premiers rôles, qui acceptent sans trop se faire prier, semble-t-il, de venir s'exprimer dans la revue : ainsi Monod, Boutroux, Durkheim, Bouglé, Rauh, et beaucoup d'autres qui lui resteront fidèles avec plus ou moins d'assiduité; enfin les jeunes intellectuels comme Simiand ou Febvre - point si nombreux d'ailleurs. La plupart d'entre eux pourraient sans doute s'exprimer ailleurs, dans cette période où se multiplient les revues scientifiques universitaires - et ils le font d'ailleurs dans L'Année sociologique, la Revue de métaphysique et de morale ou L'Année psychologique. S'ils participent aussi - et dans certains cas surtout - au premier projet de Berr, ce n'est pas nécessairement, en tout cas pas exclusivement, sur la base d'une proximité amicale ou intellectuelle, mais plutôt parce que la revue leur propose un espace libre, faiblement marqué, où l'on peut être reçu, écouté et au moins discuté. Qu'en 1903, elle ait accueilli sans hésiter le réquisitoire épistémologique de Simiand après qu'il a été présenté devant l'establishment des historiens, peut ici servir d'illustration: Simiand, après tout, n'avait pas trente ans; il n'avait pas d'œuvre personnelle visible quand il vint délivrer sa leçon de méthode ; et c'est dans la Revue plutôt que dans L'Année sociologique qu'il choisit de s'exprimer avec, on l'imagine, le souci d'être plus largement entendu ${ }^{15}$.

4. Voici donc un premier repérage qui donne un contenu au moment Berr. Un second ensemble contextuel nous renverrait plutôt du côté de ce que l'on pourrait appeler le conflit des disciplines, et dont l'intensité et les formes caractérisent si fortement les premières années du siècle. Nous nous retrouvons ici en pays partiellement connu, depuis les pistes ouvertes il y a vingt ans par V. Karady. La tension naît de l'affirmation inégale des anciennes et des nouvelles disciplines au sein de l'Université refondée, d'une part ; de l'autre, de la rivalité rapidement apparue entre les nouvelles disciplines, en particulier celles que l'on commence alors à nommer les «sciences sociales ». Il existe entre ces dernières une communauté de fait, mais aussi une communauté de projet qui est inséparable d'une double préoccupation de réforme scientifique et d'utilité sociale. De cette double préoccupation, on retrouve, bien sûr, l'expression chez les inspirateurs et chez

15. François SIMIAND, «Méthode historique et science sociale », Revue de synthèse historique, t. VI, 1903, p. 1-22, 129-137. 
les responsables de la politique universitaire de la IIIe République, mais aussi dans nombre d'initiatives privées comme l'École des hautes études sociales, par exemple.

Les disciplines « sociales " partagent-elles pour autant le même agenda épistémologique? La question est au moins posée, de façon insistante, même si elle reçoit des réponses très diverses autour de 1900. La proposition la plus nette, la plus forte, vient de Durkheim et de ses disciples, on le sait : c'est celle d'une unité épistémologique réalisée au sein de la science sociale - au singulier -, dont les démarches seraient codifiées dans les Règles de la méthode sociologique. Selon cette conception, il appartiendrait aux sciences régionales, simples provinces de ce nouvel empire, de se réformer et de reformuler leurs démarches pour se conformer aux exigences d'une véritable connaissance scientifique. Tel est, en clair, ce que Simiand recommande aux historiens en 1903, puis aux géographes à partir de 1905, aux économistes en 1909; d'autres conflits sont moins publics et non moins importants, tel celui qui opposera sociologie et psychologie jusqu'au milieu des années 20 au moins, sans parler des fronts secondaires, comme celui qui campe face à face psychologues et géographes (et qu'évoque ici L. Mucchielli) ${ }^{16}$. Ce qui est en cause derrière ces affrontements, ce sont, inséparablement, des débats de fond et des questions de prééminence entre les disciplines au sein de la nouvelle configuration intellectuelle et académique. V. Karady a fortement analysé en ce sens la stratégie minoritaire des durkheimiens et son «semi-échec ».

C'est le temps des grandes manœuvres. Dans ces mouvements compliqués, qui s'inscrivent dans le champ universitaire mais qui ne cessent de le déborder - rappelons, une fois encore, l'importance des initiatives privées ou semi privées dans le domaine des sciences sociales, dans la compétition ouverte pour leur organisation, pour leur définition, pour leur statut et leur fonction sociale -, Berr n'est intervenu que de façon discrète jusqu'à la publication de La Synthèse en histoire en 1911. C'est alors qu'apparaîtra mieux la relative cohérence de ses prises de position qu'il rationalise et qu'il durcit parfois (par exemple à l'égard du mouvement

16. Cf. Madeleine RebÉrioux, «Le débat de 1903: historiens et sociologues», in Ch.-O. Carbonell et G. Livet (éd.), Au berceau des Annales. Le milieu strasbourgeois; L'histoire en France au début du XX' siècle, Toulouse, Presses de l'IEP, 1983, p. 219-230; Roger CHARTIER, «Science sociale et découpage régional. Note sur deux débats (1820-1920), Actes de la recherche en sciences sociales, 35, 1980, p. 27-36; F. SIMIAND, «La méthode positive en sciences économiques », Revue de métaphysique et de morale, 1908, p. 889-904; du débat entre sociologues et psychologues, on prendra la mesure à travers la volumineuse bibliographie compilée par Daniel EsserTIER, Psychologie et sociologie. Essai de bibliographie critique, Paris, 1927. 
durkheimien). Jusque-là, il a essentiellement conçu son rôle comme celui d'un observateur. Il enregistre et il accueille, il met en rapport les propositions qui s'affirment de tous côtés. La Revue est un lieu où l'on peut être signalé, publié et discuté ; où l'on sait aussi pouvoir disposer d'un droit de réponse. Il est vrai que si l'on en dépouille les sommaires de façon systématique jusqu'à 1914, on a parfois plus le sentiment d'une juxtaposition que d'un véritable débat (avec quelques exceptions, comme la longue discussion entre P. Lacombe et A. D. Xénopol). Tout se passe comme si ce qui était recherché était moins l'affirmation d'une position personnelle, ou d'une ligne collective, que la recherche d'une posture arbitrale. Tel semble bien, en tout cas, le rôle que Berr occupe peu à peu. La revue est no man's land. La forte présence et l'autorité de son directeur aidant, elle devient peu à peu one man's land. Une fois de plus, La Synthèse en histoire peut être considérée comme l'aboutissement de cette évolution en ce qu'elle associe le passage en revue des propositions essentielles d'une génération intellectuelle et la lecture, les évaluations très particulières qu'en donne l'auteur en première personne, ainsi que les équilibres qu'il suggère.

Dans ces années, Berr a tout lu, il accueille tout: des propositions contraires, voire incompatibles; du bon et du moins bon aussi (avec, reconnaissons-le, une certaine tendance à se laisser abuser par les grandeurs d'établissement). Il est attentif, il est généreux, il est surtout bien informé et avide de nouveauté. Du coup, ce dont il ne parle pas prend une importance notoire parce qu'il s'agit de manières d'exception. Or, voici au moins deux noms auxquels il n'a guère prêté d'attention ${ }^{17}$, alors que la plupart des protagonistes de la scène intellectuelle sont présents : ceux de Dilthey et de Max Weber. Ils sont, objectera-t-on, plus largement absents de la réflexion française au début du siècle - et pour longtemps. Sans doute. Il reste que l'un et l'autre auraient été susceptibles, on s'en rend mieux compte aujourd'hui, de perturber et, peut-être, d'enrichir le débat français. Ils ne sont pas là, mais d'autres le sont, de vertu et d'importance inégales, qui ont été convoqués par Berr parce qu'ils s'insèrent mieux dans les espaces ouverts par ce débat, parce que, venus de l'extérieur, ils peuvent servir à en illustrer les figures possibles, parfois jusqu'à la caricature. L'éclectisme d'Henri Berr peut aussi être compris comme une entreprise de récupération et de retraitement sélectifs. Il va parfois très loin (jusqu'à chercher Benedetto Croce, qui intervient dans la revue même si le compagnonnage s'arrête bientôt). Il a aussi ses limites, inscrites dans un modèle que l'on peut tenter de préciser.

17. Ils sont, par exemple, absents de La Synthèse en histoire. 
5. On est parfois tenté de voir en Berr le symptôme et l'expression d'une réaction contre le « positivisme ». Le terme même, chacun le sait, pose problème - au point qu'il serait peut-être utile de le bannir provisoirement de notre lexique descriptif. Il est le plus souvent chargé de valeurs polémiques, en ce qu'il sert à séparer ceux qui sont encore positivistes de ceux qui ne le sont plus (et qui sont généralement connotés de façon positive). En ce sens, il relève du dictionnaire des idées reçues : "positivisme : doit être critiqué ». En outre, si l'on se situe au tournant du siècle, le mot sert à couvrir des réalités extraordinairement diverses, qui vont de l'héritage comtien à l'empiriocriticisme de Mach et à des combinaisons plus hasardeuses, comme le positivisme spiritualiste, espèce qui peut nous sembler un peu étrange avec la distance, voire oxymorique, mais bien attestée en ce temps ${ }^{18}$.

Il reste que le dernier quart du XIX ${ }^{\mathrm{e}}$ siècle a vu coexister une présence forte du positivisme et une critique globale du positivisme dont l'emblème pourrait être la thèse d'Émile Boutroux en 1874. Cette critique est la version française d'un mouvement beaucoup plus large, lui aussi parallèle à un renouvellement constant de l'affirmation positiviste. On met en cause le positivisme dominant, soit. Mais pour le remplacer par quoi ?

Si l'on se limite au débat interne aux humanités et aux sciences sociales, dans lequel on est le plus souvent en présence d'idéologies scientifiques plutôt que de positions philosophiques proprement dites, on aura tendance à répondre: par des formes différentes, plus élaborées, de positivisme. Dans la plupart des conflits qui se multiplient alors, il existe d'évidents enjeux épistémologiques qui vont peser durablement sur l'histoire des sciences sociales en France pendant une bonne partie de ce siècle ${ }^{19}$. De façon tout aussi évidente s'affrontent, en même temps, ce que l'on pourrait appeler des «styles » scientifiques et le jeu consiste, dans le même temps, à déclasser le style imputé à l'adversaire. L'offensive destructrice de François Simiand contre l'histoire «positive » et, en particulier, contre Seignobos, qui sera relayée dans les années 30-50 par la détestation tonitruante de Lucien Febvre a durablement accrédité l'image d'un Seignobos parfait représentant d'un positivisme obtus. Il y aurait beaucoup à

18. Dans une énorme littérature, deux guides réfléchis et profonds pour s'orienter dans le maquis des positivismes: Leszek KolaKowski, La Philosophie positiviste, trad. fr., Paris, Denoël, 1976; Claude NiCOLET, L'Idée républicaine en France. Essai d'histoire critique, Paris, Gallimard, 1982.

19. Faut-il rappeler que les Annales ont republié en 1960 de larges extraits du texte déjà cité de Simiand (1903), assortis de quelques lignes de F. Braudel qui se contentait d'en souligner l'actualité conservée? 
redire là-dessus, et pas seulement parce que des esprits aussi déliés que Marc Bloch ou le psychologue Ignace Meyerson ont marqué clairement leur distance d'avec cette version. Il suffit de relire l'Introduction aux études historiques (1898), ce livre tant décrié, mais surtout les articles de méthode publiés par Charles Seignobos dans les années d'avant-guerre, pour comprendre qu'il est plus subtil que l'image négative qu'a fixée de lui la polémique de 1903 ne le voudrait. Il n'est pas vrai que l'épistémologie constructiviste des durkheimiens s'oppose terme à terme à une sorte de naïveté, voire de paresse intellectuelle qui prétendrait conformer ses démarches à la configuration des faits. Est-il besoin, en outre, d'ajouter que le positivisme n'est pas ce qui sépare de la façon la plus significative la pratique de l'histoire (et des sciences sociales) selon Seignobos et la méthode sociologique que prétend imposer Simiand? Outre qu'ils partagent le même scientisme (dans des versions plus ou moins optimistes), l'insistance mise par le second à aligner les procédures des sciences sociales sur le modèle, expérimental, des sciences de la nature relève bien d'une manière de positivisme. Mais il n'y a aucune raison d'en rester à l'horizon de 1903. Dans les critiques successives du modèle durkheimien, de son caractère trop rigidement prescriptif à leurs yeux, que produiront tour à tour Henri Berr, les géographes vidaliens puis Lucien Febvre (dans $\mathrm{La}$ Terre et l'Évolution humaine), et enfin, de façon au moins implicite, les premières Annales, l'optique « positiviste »n'est jamais fondamentalement remise en cause. Ce sont, au contraire, autant de versions, plus ou moins élaborées, d'un positivisme qui n'a jamais connu de révision drastique jusqu'à une époque très récente.

Positiviste en quel sens? En ce sens que le débat des sciences sociales porte centralement sur la question et sur la nature de leur scientificité, sur les conditions qui la rendent possible. Elle tourne autour de l'opposition, science/non-science et ne laisse guère de place aux problèmes de l'interprétation tels qu'ils sont développés, au même moment (en fait, depuis les années 1830-1840), dans la tradition intellectuelle allemande. Cette réticence française va être durable, on 'le sait: l'échec de R. Aron à faire connaître les grandes lignes du débat allemand dans les années 30 en est l'un des moments significatifs, non le seul. Elle ne commence pas non plus avec le tournant du siècle: elle est sensible dès les années 1870 , lorsque la réflexion française semble évacuer un problème qui lui paraît étranger. Pourquoi? Peut-être parce que l'un des points communs à l'ensemble des «positivismes», par-delà leur diversité, c'est le privilège absolu qu'ils reconnaissent au présent dans l'histoire. Il est, provisoirement, le point de vue d'où la meilleure connaissance, la plus achevée, est possible, et c'est ce qui, précisément, permet d'évacuer les problèmes de l'interprétation. 
Sur ce point, en tout cas, rien ne sépare Simiand de Seignobos, et rien ne les sépare de l'idéaliste Berr. C'est, au contraire, le socle commun à l'auteur de La Synthèse en histoire et aux auteurs qu'il analyse et qu'il s'oppose - et qui s'opposent.

6. J'en viens ainsi à une dernière caractérisation du moment Berr, tel que je m'efforce de le définir. Il est contemporain de la «crise de la raison». Sous ce terme très générique, on évoque habituellement un enserrble de phénomènes très complexes qui couvrent les dernières années du $\mathrm{XIX}^{\mathrm{e}}$ et une bonne partie du $\mathrm{XX}^{\mathrm{e}}$ siècle. Je ne me donnerai pas le ridicule de prétendre en parler de première main devant un public où nombreux sont ceux qui sont capables de le faire bien mieux que moi. Je me contenterai d'en rappeler un certain nombre de traits qui intéressent directement le débat des sciences sociales dans le temps de leur définition intellectuelle et institutionnelle, en m'appuyant en particulier sur la thèse récente d'Enrico Castelli Gattinara qui est centralement consacrée à ce thème ${ }^{20}$.

La crise entrelace des motifs multiples et profondément liés entre eux. Elle affecte en premier lieu les «fondements » de la pensée scientifique telle qu'elle s'est constituée entre Newton et Laplace et la lecture du monde physique qui lui est associée. Elle met en cause, en second lieu, la conviction d'une unité essentielle de la science ainsi que les modèles de scientificité communément acceptés. Elle est, plus largement, crise du rationalisme et $s$ 'accompagne d'interrogations généralisées sur les formes diverses de l'activité rationnelle. Cet ébranlement touche le monde savant dans son ensemble, en suivant des étapes qu'Alexandre Koyré a résumées en distinguant, dans un premier temps, « une crise des fondements accompagnée de l'éclipse des absolus mathématiques», suivie par la double révolution de la relativité puis de la physique quantique. À cette série de secousses d'une intensité et d'une brutalité exceptionnelles, i] n'y a pas eu de réponse unifiée - bien que le débat ait été très largement international et interdisciplinaire (la Revue de synthèse historique en a été l'un des lieux, mais aussi, et de façon beaucoup plus explicite dans ces années d'avant la Première Guerre mondiale, la Revue de métaphysique et de morale dont il constitue un des axes de la réflexion collective, comme il est celui de plusieurs des grands congrès internationaux de philosophie au tournant du siècle). E. Castelli Gattinara s'est attaché à dégager les caractères originaux de la réponse française au sein

20. Enrico Castelli Gattinara, Crise de la raison, Une pensée de l'ouverture dans la France de l'entre-deux-guerres, à paraître aux éd. Vrin-EHESS, et ici même, p. 21-38. 
du concert international. Le premier est l'affirmation d'un rationalisme de fond qui, loin de la nier, «s'immerge dans la crise» et s'attache à définir une "épistémologie de temps de crise »: les noms de Poincaré, d'E. Meyerson, plus tard de Bachelard, peuvent, parmi beaucoup d'autres, servir à illustrer cette recherche. Le second, intimement associé au premier, s'emploie à trouver une issue du côté de l'histoire comme «pensée de l'ouverture ». Il ne s'agit pas seulement, tant s'en faut, de l'histoire comme discipline mais de l'histoire comme processus réflexif, de l'historicisation des énoncés et des procédures scientifiques, qui, à l'idée de système, doit permettre de substituer une perspective évolutive et dynamique. C'est le thème que reprendra Lucien Febvre dans un texte célèbre où il fait l'apologie de l'histoire, « seule capable de nous permettre, dans un monde en état d'instabilité définitive, de vivre avec d'autres réflexes que ceux de la peur».

On le voit mieux, peut-être: nous ne nous sommes éloignés de Berr qu'en apparence. Car c'est en ce point, sans doute, qu'il est au plus proche des débats scientifiques de son temps. Il est le contemporain, au sens fort du terme, de la première crise des fondements et c'est elle qui donne corps à son projet d'une synthèse générale des connaissances fondée sur une histoire. C'est lui qui donne à sa réflexion personnelle et à ses entreprises intellectuelles leur plus forte cohérence, dans ce moment et aussi dans le demi-siècle qui va suivre. Cette proposition appelle trois observations complémentaires. La «crise de la raison », c'est aussi celle du déterminisme classique, celui de Laplace qui a dominé le XIXe siècle. Pourtant, le thème en est revenu à plusieurs reprises dans nos discussions, Berr est resté un déterministe convaincu (il énoncera même en 1935 qu' «actuellement, il y a une crise du mécanisme, non pas [...] du déterminisme»), comme c'est le cas de Rey ou encore celui de Febvre. Ce qui, en revanche, paraît en cause chez eux, ce sont les modèles de causalité disponibles - et c'est mon second point. En relisant certains des textes du débat français de ces années, on est frappé par la place qu'y occupe Cournot, auquel on se réfère constamment (la Revue de métaphysique et de morale lui consacre un numéro spécial, Mentré une étude). Cela est vrai, en particulier, de Berr et de la Revue de synthèse. Pourquoi Cournot? Bien évidemment parce qu'il propose un modèle causal plus complexe et plus souple, qui paraît mieux convenir aux attentes des analystes des faits sociaux. Vidal de La Blache (mais aussi Febvre, une génération plus tard) ne l'oubliera pas. Ce qui me mène à ma troisième observation : ces modèles analytiques ne sont pas seulement des instruments conceptuels. Ils sont aussi des recours ou des ressources mobilisés par le conflit des disciplines. On le voit bien dans le cas de la géographie vidalienne face à l'épistémologie prescriptive de Durk- 
heim et de ses disciples ${ }^{21}$. On le verra, de façon qui n'est pas toujours explicite, dans les premières Annales et dans leur souci de mettre au jour, plutôt que des « lois », des réseaux d'interrelations et d'interdépendance entre les faits les plus complexes possible. On l'a vu aussi, dès 1911, dans les schémas qu'esquisse La Synthèse en histoire.

7. Ce qui retient d'abord, chez Berr, c'est l'originalité de l'homme et celle du projet, qui paraît parfois confiner à l'excentricité, en tout cas à l'obsession. En témoignent un lexique et une rhétorique, l'inflexible énergie d'un entrepreneur obnubilé par l'organisation du savoir, son obstination tout au long d'un demi-siècle. Aussi bien peut-il sembler paradoxal de prétendre dissoudre le personnage dans un contexte sur lequel il se détache aussi nettement; paradoxal aussi de suggérer que le «moment Berr» s'identifie aux années d'avant-guerre alors que son action va continuer pendant un demisiècle. On espère avoir montré que l'originalité de Berr ressort d'autant mieux, et qu'elle a été d'autant mieux entendue qu'elle nouait ensemble, dans un style très particulier, il est vrai, des thèmes, des lectures, des préoccupations qui traversaient le monde intellectuel. Berr les a orchestrés à sa manière, selon une formule institutionnelle et en mettant en œuvre des ressources relationnelles qui lui étaient propres, mais il pouvait être entendu de tous ceux qui comptaient. Le moment Berr s'achève-t-il avec la Première Guerre mondiale? Il est vrai que son grand livre est de 1911, que la conception et le plan de sa collection "L'Évolution de l'humanité » étaient arrêtés avant 1914; il est vrai aussi que les créations institutionnelles les plus importantes, les plus visibles aussi, viendront dans les années 20 avec le Centre de synthèse, la Fondation, les Semaines - et que rien ne nous oblige à penser que le temps de la croissance institutionnelle est nécessairement celui du déclin de la pensée. Mais ces institutions vont fonctionner dans un monde profondément renouvelé alors que le projet intellectuel de Berr, lui, est demeuré substantiellement identique. Il s'est plutôt durci, avec le passage de la synthèse historique à l'ambition d'une synthèse générale alors même que le problème de l'unité des sciences est plus durement posé. Dans les années 20-30, sa capacité d'initiative reste forte, elle est même spectaculaire; elle est portée en outre par une vraie reconnaissance publique. Mais on a souvent le sentiment que son rôle s'est réduit à l'activité institutionnelle, assurant la mise en scène de confrontations dont les arguments et les enjeux lui échappent en partie. C'est alors le temps du système Berr. Mais le moment Berr est peut-être passé.

21. Vincent Berdoulay, La Formation de l'École française de géographie, Paris, Publications du Comité des travaux historiques et scientifiques, Mémoires de la Section de géographie, 11, Bibliothèque nationale, 1981 (en particulier le chapitre consacré à l'épistémologie vidalienne). 